\title{
Uso dei calciomimetici nell'iperaparatiroidismo secondario
}

\section{P. Messa}

U.O. di Nefrologia, Dialisi e Trapianto Renale, Ospedale Maggiore-Policlinico, Mangiagalli e Regina Elena, Fondazione IRCCS, Milano

\section{Introduzione}

L’iperparatiroidismo secondario (IPTS) inizia sin dalle prime fasi dell'insufficienza renale cronica (IRC), riconoscendo numerosi fattori causali, solo in parte noti, tra i quali i prinicipali sono elencati nella Tabella I (1-3).

Da lungo tempo l'IPTS dell'IRC è riconosciuto come il fattore causale di primaria importanza nel determinare la patologia ossea del paziente uremico e delle sue principali conseguenze cliniche: il dolore e le fratture. In aggiunta a questo, nell'ultimo decennio si sono andate accumulando evidenze sperimentali e in parte anche cliniche che hanno suggerito che l'IPTS e le alterazioni del metabolismo minerale a esso associate possano avere parte nel contribuire all'elevata incidenza delle calcificazioni vascolari, della morbilità e della mortalità cardio-vascolare che caratterizza il paziente con IRC, sin dalle prime fasi della malattia (4-6). Queste considerazioni hanno spinto i Nefrologi a rinominare quella che un tempo veniva riduttivamente definita Ostedistrofia Uremica in una sindrome clinica più complessa, se pure ancora non del tutto definita, a cui si è deciso di dare la denominazione di malattia renale cronica e disordini del metabolismo minerale (CKDBMD) (7). L'approccio terapeutico ottimale di questa nuova entità clinica dovrebbe pertanto tenere in considerazione non solo la prevenzione dello sviluppo della patologia ossea correlate all'IPTS, ma anche del processo di calcificazione vascolare e degli eventi clinici a esso associati, attraverso uno stretto controllo non solo dei livelli di PTH, ma anche di quelli del calcio e del fosfato, come suggerito dalle linee guida internazionali provenienti dalla National Kidney Foundation-Kid-
ney/Dialysis Outcomes Quality Initiative (NKFK/DOQI) (8).

Per molto tempo i metaboliti della vitamina $\mathrm{D}$ e i chelanti del fosfato hanno rappresentato il principale, se non l'unico, mezzo terapeutico per il controllo dell'IPTS del paziente con IRC. È comunque noto che tale terapia, sebbene efficace nel controllo del PTH, si sia dimostrata non altrettanto efficace sul controllo contemporaneo della calcemia e della fosforemia (9).

\section{Scoperta e caratterizzazione del Recettore Sensibile al Calcio (CaSR)}

Che numerosi sistemi cellulari siano in grado di rispondere direttamente a modificazioni della calcemia è noto da tempo, ma fu solo un ventennio fa che Brown et al, dimostrarono la presenza di un sensore per il calcio sulla membrane delle cellule paratiroidee in grado di modulare la secrezione del PTH (10). A pochi anni di distanza gli stessi ricercatori furono in grado di clonare il gene che trascriveva per il CaSR (11). Il CaSR appartiene alla famiglia delle G-proteine ed è in grado di rispondere non solo alle variazioni della concentrazione di calcio, ma anche di numerose altre sostanze cationiche, organiche e inorganiche (12).

Al CaSR appartengono 3 domini: il primo amino-terminale extracitoplasmatico che interagisce con gli agonisti; il secondo intramembranoso che contiene i siti responsabili della dimerizzazione e di interazioni con $\mathrm{i}$ modulatori allosterici; il terzo intracitoplasmatico contenente il terminale carbossilico e responsabile della trasmissione del segnale all'interno della cellula (13). L'attivazione del CaSR porta alla fosforilazione di diffe- 
renti proteine $\mathrm{G}\left(\mathrm{Gi}, \mathrm{Gq}, \mathrm{G}_{12 / 13}\right)$, a cui segue l'attivazione di una serie di mediatori (adenyl cyclase, Phospholipase-A, -C, D, Inositol-Tri-Phosphate kinase, PKC, JNK, ERK ecc.) differenti nei vari sistemi cellulari in cui il CaSR è espresso, che conducono a eventi organo o tessuto specifici (14).

Il CaSR è espresso non solo a livello di quelle cellule coinvolte nella secrezione degli ormoni calciotropi (cellule paratiroidee, cellule C della tiroide) o dei sistemi cellulari responsabili del trasporto del calcio (tubuli renali, enterociti, osteoblasti/osteociti/ condrociti), ma anche in numerosi altri tessuti nei quali il suo ruolo è ancora poco definito (15).

L'attivazione del CaSR sulle cellule paratiroidee indotta dall'aumento della concentrazione del calcio induce un consensuale incremento della concentrazione del calcio intra-citosolico a cui consegue nell'arco di secondi o minuti una diminuzione del rilascio del PTH preformato dalle vescicole secretorie. Se la stimolazione del CaSR dura più a lungo, segue una riduzione della trascrizione e verosimilmente della stabilità dell'mRNA del PTH, nell'arco di ore o giorni. Una più prolungata stimolazione del recettore può condurre nell'arco di mesi o anni a una inibizione dei processi proliferativi e forse a un incremento dell'apoptosi delle cellule paratiroidee (16).

L'attivazione del CaSR a livello delle cellule tubulari renali (glomerulo, tubulo prossimale, distale, ansa di Henle, tubulo collettore) induce una riduzione del riassorbimento del calcio, sodio e dell'acqua $(17,18)$.
È ormai dimostrato che il CaSR è espresso anche a livello delle cellule del tessuto osseo e può mediare direttamente la risposta alle variazioni del calcio, indipendentemente dal PTH e dalla vitamina $\mathrm{D}(19,20)$.

E argomento di dibattito se le variazioni dell'espressione e/o della funzionalità del CaSR possano costituire una causa dello sviluppo dell'IPTS dell'IRC.

Sebbene alcune evidenze sperimentali suggeriscano che una riduzione dell'espressione del CaSR sulle cellule paratiroidee e le conseguenti variazioni del loro set-point e sensibilità al calcio possano avere un ruolo nello sviluppo dell'IPTS in corso di IRC $(21,22)$, questi eventi verosimilmente accadono in stadi più avanzati della malattia e non caratterizzano pertanto le prime fasi di sviluppo dell'IPTS $(23,24)$.

\section{I calciomimetici}

Tutti gli agonisti del CaSR, se si eccettua il calcio stesso, possono in un certo senso essere denominati calciomimetici di tipo I. È chiaro comunque che l'interesse maggiore si è sviluppato intorno all'utilizzazione di alcuni nuovi composti (calciomimetici di tipo II) che, interagendo con i domini intramembranosi del CaSR, inducono delle modificazioni steriche a cui consegue una riduzione della soglia di attivazione da parte degli agonisti (25). Numerosi sono stati i composti che avevano queste caratteristiche, ma tra tutti Cinacalcet

\section{TABELLA I - ALCUNI DEI FATTORI SUGGERITI COME CAUSALI NELLA GENESI DELL'IPTS DELL'IRC}

Riduzione della biodisponibilità ed efficacia della vitamina $D$

Cause:

- Ridotta sintesi di 1,25-di-hydroxyvitamin D per riduzione della massa renale e aumento dei livelli di FGF-23

- Ridotta disponibilità del precursore (25-hydroxyvitamin D)

- Ridotta espressione dei VDR sulle cellule target della vitamina D

Ridotta concentrazione di calcio

Cause:

- Ridotto assorbimento intestinale secondario al deficit di vitamina D

- Ridotta rimozione ossea del calcio secondaria alla resistenza all'effetto del PTH e della vitamina D

- Aumento della fosfatemia

Aumento dei livelli di fosfato

Cause:

- Ridotta escrezione urinaria

- Ridotta capacità di tamponamento dell'incremento dei fosfati da parte dell'osso 
$\mathrm{HCl}$ si è dimostrato quello con le caratteristiche farmacocinetiche e farmacodinamiche meglio rispondenti alle attese per una possibile applicazione clinica. Cinacalcet è l'R-enantiomero dell'AMG 073 ed è stato dimostrato sia in studi in vitro che in vivo inibire la secrezione del PTH mediata da variazione della concentrazione del $\mathrm{Ca}^{2+}(26)$.

La biodisponibilità di una dose orale di Cinacalcet è intorno al 70-75\%, raggiungendo una $\mathrm{C}_{\max }$ circa $2-6$ ore dopo l'assunzione del farmaco. Sia la $\mathrm{C}_{\max }$ che l'AUC $\mathrm{C}_{0-24}$ aumentano proporzionalmente alla dose somministrata sino a una dose massima di $200 \mathrm{mg}$ oltre la quale non si osservano ulteriori aumenti della concentrazione ematica $(7,28)$. È stata inoltre dimostrata una relazione molto stretta tra le concentrazioni ematiche del farmaco e l'entità degli effetti inibitori sulla secrezione di $\mathrm{PTH}$, con un effetto massimale che viene raggiunto a concentrazioni di $20 \mathrm{ng} / \mathrm{mL}$ (29). È importante ricordare che la biodisponibilità del farmaco è influenzata dal momento dell'assunzione: se assunto in associazione al cibo il suo assorbimento può infatti essere incrementato sino al $70 \%$ in più rispetto all'assunzione a digiuno (30).

Cinacalcet è prevalentemente metabolizzato a livello epatico principalmente dal CYP2D6, CYP1A2 e dal CYP3A4. Nei pazienti con danno funzionale epatico severo è pertanto da attendersi una biodisponibilità del farmaco aumentata (27). Al contrario, non è stata dimostrata alcuna influenza della funzione renale, dei differenti trattamenti dialitici e del trapianto di rene sul metabolismo e/o sulla clearance di Cinacalcet $(31,32)$.

\section{Utilizzo clinico di Cinacalcet nell'IPTS dell'IRC}

Cinacalcet è stato approvato sia dalla FDA che dall'EMEA per il trattamento dell'IPTS associato all'IRC solo per i pazienti con insufficienza renale cronica in trattamento dialitico. In ogni caso la sua utilizzazione è stata proposta anche per gli stadi di IRC predialitici e per l'IPTS persistente dopo il trapianto di rene (33).

\section{Cinacalcet nel trattamento dell'IPTS nei pazienti in trattamento dialitico}

I primi studi clinici, effettuati in piccole coorti di pazienti in dialisi, avevano dimostrato che Cinacalcet era efficace nel ridurre non solo i livelli di PTH, ma anche quelli della calcio e in parte anche quelli del fosfato $(26,34-36)$.

Nel 2004 Block e un gruppo di altri autori pubblicaro- no i risultati di uno studio multicentrico (multi continentale) randomizzato, a doppio cieco, che metteva a confronto i risultati di Cinacalcet aggiunto a una terapia standard con un gruppo in cui alla terapia in atto si aggiungeva del placebo (37). Lo studio che comprendeva 741 pazienti in dialisi seguiti per 26 settimane, dimostrò che il $43 \%$ dei pazienti trattati con Cinacalcet contro solo il 5\% dei pazienti nel gruppo placebo raggiungeva il target prefissato per il PTH (150-250 $\mathrm{pg} / \mathrm{mL}$ ). In effetti questo target era lievemente differente rispetto a quello suggerito dalle linee guida $\mathrm{K} / \mathrm{DOQI}$. Tale discrepanza era in parte spiegata dal fatto che lo studio era stato disegnato poco prima della pubblicazione di queste linee guida. Un altro importante risultato era quello di una sensibile riduzione del calcio $(6.4 \%)$ e del fosfato $(8.4 \%)$ nei pazienti trattati con Cinacalcet, rispetto a una tendenza a un incremento degli stessi parametri nel gruppo di controllo. In tutti questi studi venivano riportati come effetti collaterali più frequenti legati all'uso di Cinacalcet quelli relativi all'apparato gastroenterico (nausea, vomito), oltre all'ipocalcemia. Si ritiene che l'ipocalcemia secondaria all'uso dei calciomimetici sia la conseguenza della riduzione dei livelli di PTH, a cui consegue la riduzione del riassorbimento osseo di calcio mediato dal PTH stesso. Non è però da sottovalutare il possibile ruolo degli effetti di Cinacalcet sulle cellule intestinali che esprimono come è noto il CaSR, alla cui stimolazione potrebbe conseguire anche una riduzione dell'assorbimento del calcio. Peraltro non può neppure essere escluso che l'effetto ipocalcemizzante del calciomimetico sia una conseguenza dell'effetto diretto del farmaco sulle cellule ossee anch'esse fornite del recettore specifico per il calcio. I disturbi gastroenterici potrebbero invece essere almeno in parte conseguenza dell'effetto stimolatore di Cinacalcet sulla secrezione acidica gastrica.

Uno studio successivo dimostrò che Cinacalcet manteneva la sua efficacia nel controllo del PTH, oltre che del calcio e del fosfato, anche a 3 anni dall'inizio del trattamento (38).

Questi risultati venivano peraltro ribaditi da una metanalisi di tutti gli studi prodotti sull'argomento (39). Gli studi successivi hanno quindi affrontato il problema di come ottimizzare l'uso di Cinacalcet e in particolare di come combinarne l'uso con quello della vitamina D. Lo studio OPTIMA ha avuto precisamente lo scopo di fornire un algoritmo di utilizzazione di Cinacalcet in associazione alla terapia standard (vitamina D + chelanti del fosfato) confrontandone l'efficacia sul controllo dei parametri bioumorali in raffronto a un uso della terapia standard effettuato secondo la prassi 
clinica in atto. Ancora una volta i risultati confermavano la superiorità dell'aggiunta di Cinacalcet nel raggiungimento dei target biochimici, con una discreta riduzione $(-22 \%)$ della dose dei metaboliti della vitamina D utilizzati in associazione (40).

Due studi successivi, il TARGET (41) e l'ACHIEVE (42), hanno invece testato l'efficacia di Cinacalcet in combinazione con dosi minimizzate dei preparati vitaminici $\mathrm{D}$, in raffronto a un uso libero e flessibile di tali preparati, senza associazione con il calciomimetico. Entrambi gli studi hanno dimostrato che i pazienti in Cinacalcet raggiungevano i target biochimici in maggiore percentuale rispetto al gruppo trattato con sola vitamina $\mathrm{D}$.

La conclusione comune di tutti questi studi era che, a fronte di una maggiore probabilità di raggiungere i target K/DOQI per i parametri del metabolismo minerale, Cinacalcet, se aggiunto alla terapia standard permette un sostanziale effetto di risparmio sulle dosi di vitamina $\mathrm{D}$.

Sulla base di questi risultati, ma anche di altre proposte terapeutiche alternative, si sono andate sviluppando tre principali "linee di pensiero" sulle modalità di approccio terapeutico dell'IPTS dell'IRC.

La prima favorisce l'uso della vitamina D come farmaco di prima linea, con l'uso di Cinacalcet solo come terapia di salvataggio, in caso di forme gravi e non risolvibili di IPTS. La seconda linea di pensiero consiglierebbe al contrario Cinacalcet come primo farmaco da utilizzare, possibilmente, ma non necessariamente associato all'uso della vitamina D. La terza modalità suggerita è quella di una combinazione di piccole dosi dei due farmaci ove possible sin dalle prime fasi, modulandone il dosaggio in funzione delle variazioni non solo del PTH, ma anche della calcemia e fosforemia. I pro e contro di queste tre tipologie di proposta sono ancora oggetto di dibattito (43-45).

Comunque sia, tutti questi studi hanno semplicemente dimostrato come le terapie proposte possono più o meno soddisfare la richiesta di un raggiungimento dei parametri bioumorali. Se questo migliore controllo metabolico si traduca in un reale miglioramento degli outcome clinici (fratture, numero di paratiroidectomie, morbilità e mortalità totale e cardio-vascolare) è lontano dall'essere stato sino a ora dimostrato.

Alcuni studi, peraltro condotti in piccoli gruppi di pazienti o basati su analisi post-hoc di trial precedenti, suggerirebbero un qualche miglioramento dei parametri istologici ossei (46) e del contenuto minerale osseo $(47,48)$, una riduzione dell'incidenza di fratture, della necessità di sottoporsi a paratiroidectomia e di ospedalizzazione (49).
In aggiunta a questo, alcuni studi sperimentali e alcune recenti osservazioni cliniche sembrerebbero suggerire l'ipotesi che l'uso di Cinacalcet potrebbe indurre una regressione dell'iperplasia paratiroidea $(50,51)$.

Un altro argomento intorno al quale sta crescendo un interesse sempre maggiore è quello che riguarda i potenziali effetti del calciomimetico sul processo delle calcificazioni vascolari. Alcuni studi sperimentali sembrerebbero suggerire che i calciomimetici possono inibire il processo di calcificazione associato all'uremia $(52,53)$. Inoltre, i calciomimetici sembrerebbero avere un effetto protettivo nei confronti dell'effetto pro-calcificante che si osserva con l'uso dei metaboliti della vitamina $\mathrm{D}$ sia nella parete vascolare che nel muscolo cardiaco del ratto reso uremico (54). D'altra parte, un recente studio condotto in pazienti in dialisi ha evidenziato un incremento dell'Osteoprotegerina (OPG) e una riduzione dei livelli di Fetuina-A dopo 1 anno di trattamento con Cinacalcet (55). Dal momento che sia l'aumento dei livelli di OPG che ridotte concentrazioni di Fetuina-A sono state associate con aumentato rischio di sviluppare calcificazioni vascolari, questi risultati preliminari sembrerebbero suggerire un potenziale aumento di tale rischio nei pazienti che si sottopongano a tale trattamento. É però necessario specificare che, data la complessità del processo di calcificazione della parete vascolare, le alterazioni segnalate potrebbero rappresentare un riequilibrio compensatorio di alcuni tra $\mathrm{i}$ tanti fattori coinvolti, in presenza di una riduzione del rischio complessivo di calcificazione vascolare.

Uno studio prospettico, randomizzato e in cieco che è in corso, ci darà probabilmente la risposta definitiva sull'eventuale efficacia di Cinacalcet nel ridurre la morbidità e la mortalità cardiovascolare nei pazienti in dialisi, in confronto con la terapia standard (56).

\section{Cinacalcet nel trattamento dell'IPTS dei pazienti con IRC in fase pre-dialitica}

Il razionale per l'uso di Cinacalcet sin dai primi stadi della malattia renale cronica risiede nella considerazione che l'IPTS inizia già in queste fasi precoci. Inoltre la terapia sino a ora utilizzata non ha impedito in modo sostanziale la riduzione della progressione verso l'iperplasia ghiandolare autonoma.

Uno studio effettuato in pazienti con IRC, non ancora in trattamento dialitico, aveva dimostrato un'efficacia di Cinacalcet nel ridurre i livelli di PTH (57). Questo effetto si era però dimostrato associato con una sensibile riduzione della calcemia e un discreto 
incremento dei livelli di fosfatemia. In particolare questo secondo effetto ha indotto non poche perplessità sull'uso di tale farmaco nelle fasi di IRC pre-dialitiche, dal momento che innalzamenti anche modesti dei livelli di fosfatemia, ben all'interno dei limiti dei valori considerati normali, sono stati associati a una più elevata mortalità nei pazienti con IRC negli stadi pre-dialitici (58). Inoltre, è da considerare che non è stata evidenziata alcuna variazione della curva di risposta del PTH alle variazioni di calcio in queste fasi più precoci della malattia renale, rendendo poco plausibile il ricorso a un intervento farmacologico che miri a correggere un'attività ancora normale (24). Sulla base di tutte queste considerazioni, si dibatte ancora se queste fasi iniziali dell'IPTS debbano essere o meno un terreno di utilizzo dei calciomimetici $(59,60)$.

\section{Cinacalcet per il trattamento dell'IPTS persistente dopo trapianto renale}

Il trapianto renale corregge la maggior parte dei sintomi legati allo stato uremico. Nonostante ciò, una certa quota di IPTS residuale permane anche a lunga distanza dal trapianto e non infrequentemente è associato a un grado variabile di ipercalcemia $(61,62)$. Inoltre, si ritiene che sia gli elevati livelli di PTH che quelli di calcio possano avere un impatto negativo e sul già precario trofismo osseo di questi pazienti che persino della funzione dell'organo trapiantato (63). Su questi presupposti, l'uso di Cinacalcet è stato proposto anche per il trattamento dell'IPTS persistente dopo trapianto renale, in particolare se associato a ipercalcemia.

Dal momento che Cinacalcet non è stato ancora approvato per l'uso in questi pazienti, i dati sino a ora disponibili su questo argomento provengono da studi condotti su piccole coorti di pazienti, senza gruppo di controllo e con follow-up relativamente brevi. Ciononostante tutti questi studi dimostrano in modo abbastanza omogeneo che Cinacalcet induce una variabile riduzione del PTH e dei livelli di calcemia, con un consistente incremento della fosfatemia e del contenuto minerale osseo $(64,65)$. Di fatto, l'aumento della fosfatemia, evento non desiderato nell'IRC predialitica, può essere considerato al contrario un effetto positivo nel paziente trapiantato, che spesso presenta valori patologicamente ridotti di fosfatemia che possono impattare negativamente sul trofismo dell'osso, contribuendo ad incrementarne la demineralizzazione.

Inoltre non è stata segnalata alcuna interferenza di
Cinacalcet con il metabolismo dei principali farmaci utilizzati nei regimi immunosoppressivi (66).

Sebbene questi risultati provengano da studi criticabili metodologicamente, suggeriscono comunque che potrebbe essere proponibile l'uso di Cinacalcet in tale condizione.

\section{Conclusioni}

Cinacalcet rappresenta una nuova ed efficace opzione terapeutica per il trattamento dell'IPTS dell'IRC. Se non vi sono dubbi sul fatto che tale farmaco sia efficace nell'aumentare le probabilità del raggiungimento dei target per i parametri bioumorali del metabolismo minerale, rimangono ancora da chiarire numerosi punti. Innanzitutto, il reale vantaggio di utilizzarlo in fasi molto precoci della malattia rimane da dimostrare.

In ogni caso, il principale quesito che rimane aperto per questo come per ogni altro farmaco utilizzato in tale contesto riguarda la possibilità di dimostrare che l'aumento della qualità del controllo dei parametri bioumorali si traduca di fatto anche in un miglioramento degli outcome clinici.

\section{Ringraziamenti}

Questo studio è stato supportato dal grant "Progetto Glomerulonefriti" in memoria di Pippo Neglia.

Indirizzo dell'Autore:

Piergiorgio Messa, MD

Nefrologia, Dialisi e Trapianto

Padiglione Croff

Ospedale Maggiore-Policlinico

Via Commenda, 15

20122 Milano

pmessa@policlinico.mi.it 


\section{Bibliografia}

1. Levin A, Bakris GL, Molitsh M, et al Prevalence of abnormal serum vitamin D, PTH, calcium, and phosphorus in patients with chronic kidney disease: results of the study to evaluateearly kidney disease. Kidney Int 2007; 71: 31-8.

2. Moranne O, Froissart M, Rossert J, et al. Timing of onset of CKD-related metabolic complications. J Am Soc Nephrol 2009; 20: 164-71.

3. Goodman WG, Quarles LD. Development and progression of secondary hyperparathyroidism in chronic kidney disease: lessons from molecular genetics. Kidney Int 2008;74: 276-88.

4. Ganesh SK, Stack AG, Levin NW, et al. Association of elevated serum $\mathrm{PO} 4$, Ca xPO4 product, and parathyroidhormone with cardiac mortality risk in chronic hemodialysis patients. J Am Soc Nephrol 2001; 12: 2131-8.

5. Block GA, Port FK. Re-evaluation of risks associated with hyperphosphatemia and hyperparathyroidism in dialysis patients: recommendations for a change in management. Am J Kidney Dis 2000; 35:1226-37.

6. Block G, Port FK. Calcium phosphate metabolism and cardiovascular disease in patients with chronic kidney disease. Semin Dial 2003; 16: 140-7.

7. Moe SM, Drüeke T, Lameire N, Eknoyan G. Chronic kidney disease-mineral-bone disorder: a new paradigm. Adv Chronic Kidney Dis 2007; 14(1): 3-12.

8. National Kidney Foundation: K/DOQI clinical practice guidelines on bone metabolism and disease. Am J Kidney Dis 2003; 42 (Suppl 3): S1-201.

9. Young EW, Albert JM, Satayathum S, et al. Predictors and consequences of altered mineral metabolism: the Dialysis Outcomes and Practice Patterns Study. Kidney Int 2005; 67: 1179-87.

10. Brown EM. Extracellular $\mathrm{Ca} 2+$ sensing, regulation of parathyroid cell function, and role of $\mathrm{Ca} 2+$ and other ions as extracellular (first) messenger. Physiol Rev 1991; 71: 371-411.

11. Brown EM, Gamba G, Riccardi D, et al. Cloning and characterization of an extracellular $\mathrm{Ca}(2+)$-sensing receptor from bovine parathyroid. Nature 1993; 366: 57580.

12. Chang W, Shoback D. Extracellular Ca2+ sensing receptors - an overview. Cell Calcium 2004; 35: 183-96.

13. Bai M. Structure-function relationship of the extracellular calcium-sensing receptor. Cell Calcium 2004; 35: 197-207.

14. Ward, Donald T. Calcium receptor-mediated intracellular signalling. Cell Calcium 2004; 35: 217-28.

15. Coburn JW, Elangovan L, Goodman WG, Frazao JM. Calcium-sensing receptor and calcimimetic agents. Kidney Int 1999; 73 (Suppl): S52-8.
16. Brown EM. Calcium receptor and regulation of parathyroid hormone secretion. Rew Endocrine and Metab Dis 2000; 1: 307-15.

17. Ba J, Friedman PA. Calcium-sensing receptor regulation of renal mineral ion transport. Cell Calcium 2004; 35: 229-37.

18. Gamba G, Friedman PA. Thick ascending limb: the $\mathrm{Na}(+): \mathrm{K}(+): 2 \mathrm{Cl}(-)$ co-transporter, NKCC2, and the calcium-sensing receptor, CaSR. Pflugers Arch 2008 Nov 4. [Epub ahead of print].

19. Dvorak MM, Riccardi D. $\mathrm{Ca}(2+)$ as an extracellular signal in bone. Cell Calcium 2004; 35: 249-55.

20. Chang W, Tu C, Chen TH, Bikle D, Shoback D. The extracellular calcium-sensing receptor (CaSR) is a critical modulator of skeletal development. Sci Signal 2008; 1(35): ra1

21. Brown EM, Wilson RE, Eastman RC,Pallotta J, Marynick P. Abnormalregulation of parathyroidhormone release by calcium in secondary hyperparathyroidism due to chronic renal failure. J Clin Endocrinol Metab 1982; 54: 172-9.

22. Brown AJ, Ritter CS, Finch JL, Slatopolsky EA. Decreased calcium-sensing receptor extression in hyperplastic parathyroid glands of uremic rats: role of dietary phosphate. Kidney Int 1999; 55: 1284-92.

23. Malberti F, Faina M, Imbasciati E. The PTH-calcium curve and the set point of calcium in primary and secondaryhy perparathyroidism. Nephrol Dial Transplant 1999; 14: 2398-406.

24. Messa P, Vallone C, Mioni G, Geatti O, Turrin D, Passoni N, Cruciatti A. Direct in vivo assessment of parathyroid hormone-calcium relationship curve in renal patients. Kidney Int 1994; 46:1713-20.

25. Nemeth EF, Steffey ME, Hammerland LG, et al. Calcimimetics with potent and selectiveactivity on the parathyroid calcium receptor. Proc Natl Acad Sci USA 1998; 95: 4040-5-

26. Goodman WG, Frazao JM, Goodkin DA, et al. A calcimimetic agent lowers plasma parathyroid hormone levels in patients with secondary hyperparathyroidism. Kidney Int 2000; 58: 436-45.

27. Kumar GN, Sproul C, Poppe L, et al. Metabolism and disposition of calcimimetic agent cinacalcet $\mathrm{HCl}$ in humans and animal models. Drug Metab Dispos 2004; 32: 1491-500.

28. Harris RZ, Padhi D, Marbury TC, Noveck RJ, Salfi M, Sullivan JT. Pharmacokinetics, pharmacodynamic, and safety of cinacalcet hydrochloride in hemodialysis patients at doses up to $200 \mathrm{mg}$ once daily. Am J Kidney Dis 2004; 44: 1070-76.

29. Balfour JAB, Scott LJ. Cinacalcet Hydrochloride. Drugs 2005; 65: 271-81.

30. Padhi D, Salfi M, Harris RZ. The pharmacokinetics of Cinacalcet are unaffected following consumption of 
high- and low-fat meals. Am J Ther 2007; 14: 235-40.

31. Lindberg JS, Culleton B, Wong G, et al. Cinacalcet $\mathrm{HCl}$, an oral calcimimetic agent for the treatment of secondary hyperparathyroidism in hemodialysis and peritoneal dialysis: a randomized, double-blind, multicenter study. J Am Soc Nephrol 2005; 16: 800-07.

32. Serra AL, Braun SC, Starke A, et al. Pharmacokinetics and pharmacodynamics of Cinacalcet in patients with hyperparathyroidism after renal transplantation. Am J Transplant 2008; 8: 803-10.

33. Evenepoel P. Calcimimetics in chronic kidney disease: evidence, opportunities and challenges. Kidney Int 2008; 74: 265-75.

34. Goodman WG, Hladik GA, Turner SA, et al: The calcimimetic agent AMG 073 lowers plasma hormone levels in hemodialysis patients with secondary hyperparathyroidism. J Am Soc Nephrol 2002; 13: 1017-24.

35. Quarles LD, Sherrard DJ, Adler S, et al.The calcimimetic AMG 073 as a potential treatment for secondary hyperparathyroidism of end-stage renal disease. J Am Soc Nephrol 2003; 14: 575-83.

36. Lindberg JS, Moe SM, Goodman WG, et al. The calcimimetic AMG 073 reduces parathyroid hormone and calcium $\mathrm{x}$ phosphorus in secondary hyperparathyroidism. Kidney Int 2003; 63: 248-54.

37. Block GA, Martin KJ, de Francisco ALM, et al. Cinacalcet for secondary hyperparathyroidism in patients receiving hemodialysis. New Eng J Med 2004; 350: 1516-25.

38. Moe SM, CunninghamJ, Bommer J, et al. Long-term treatment of secondary hyperparathyroidism with the calcimimetic cinacalcet $\mathrm{HCl}$. Nephrol Dial Transplant 2005; 20: 2186-93.

39. Strippoli GFM, Palmer S, Tong A, Elder G, Messa P, Craig JC. Meta-analysis of biochemical and patient-level effects of calcimimetic therapy. Am J Kidney Dis 2006; 47: 715-26.

40. Messa P, Macario F, Yagoob M, et al. The optima study: assessing a new cinacalcet (Sensipar/Mimpara) treatment algorithm for secondary hyperparathyroidism. Clin J Am Soc Nephrol 2008; 3: 36-45.

41. Block GA, Zeig S, Sugihara J, Chertow GM, Chi EM, Turner SA, Bushinsky DA; TARGET Investigators. Combined therapy with cinacalcet and low doses of vitamin D sterols in patients with moderate to severe secondary hyperparathyroidism. Nephrol Dial Transplant 2008; 23(7): 2311-8.

42. Fishbane S, Shapiro WB, Corry DB, et al. Cinacalcet $\mathrm{HCl}$ and concurrent low-dose vitamin D improves treatment of secondary hyperparathyroidism in dialysis patients compared with vitamin D alone: the ACHIEVE study results. Clin J Am Soc Nephrol 2008; 3 (6): 1718-25.

43. Cannella G, Messa P. Therapy of secondary hyper- parathyroidism to date: vitamin $\mathrm{D}$ analogs, calcimimetics or both? J Nephrol 2006; 19(4): 399-402.

44. Drüeke TB, Ritz E. Treatment of secondary hyperparathyroidism n CKD patients with Cinacalcet and /or Vitamin D Derivatives. Clin J Am Soc Nephrol 2009; 4: 234-41.

45. Wetmore JB, Quarles LD. Calcimimetics or vitamin D analogs for suppressing parathyroid hormone in endstage renal disease: time for a paradigm shift? Nature Clin Pract Nephrol 2009; 5: 24-33.

46. Malluche HH, Moner-Faugere MC, Wang G, et al. An assessment of cinacalcet $\mathrm{HCl}$ effects on bone histology in dialysis patients with secondary hyperparathyroidism. Clin Nephrol 2008; 69: 269-77.

47. Lien YHH, Silva AL, Whittman D. Effects of Cinacalcet on bone mineral density in patients with secondary hyperparathyroidism. Nephrol Dial Transplant 2005; 20: 1232-7.

48. Ureña Torres P. Clinical experience with cinacalcet HCl. Nephrol Dial Transplant 2004; 19 (Suppl 5): v27-v33.

49. Cunningham J, Danese M, Olson K, Klassen P, Chertow GM. Effects of the calcimimetic cinacalcet $\mathrm{HCl}$ on cardiovascular disease, fracture, and health-related quality of life in secondary hyperparathyroidism. Kidney Int 2005; 68:1793-800.

50. Colloton M, Shatzen E, Miller G, et al. Cinacalcet $\mathrm{HCl}$ attenuates parathyroid hyperplasia in a rat model of secondary hyperparathyroidism. Kidney Int 2005; 67: 467-76.

51. Meola M, Petrucci I, Barsotti G. Long-term treatment with cinacalcet and conventional therapy reduces parathyroidhyperplasia in severe secondary hyperparathyroidism. Nephrol Dial Transplant 2009; 24(3): 982-9.

52. Kawata T,Nagano N, Obi M, et al. Cinacalcet suppresses calcification of the aorta and heart in uremic rats. Kidney Int 2008; 74: 1270-7.

53. Ivanovski O, Nikolov IG, Joki N, et al. The calcimimetic R-568 retards uremia-enhanced vascular calcification and atherosclerosis in apolipoprotein $\mathrm{E}$ deficient (apo/- ) mice. Atherosclerosis 2009; I n press.

54. Lopez J, Mendoza FJ, Agulera-Tejero E, et al. The effect of calcitriol, paricalcitol, and a calcimimetic on extraosseous calcifications in uremic rats. Kidney Int 2008; 73: 300-07.

55. Messa P, Alberti L, Como G, et al. Calcimimetic increases osteoprotegerin and decreases fetuin-A levels in dialysis patients. Nephrol Dial Transplant 2007; 22: 2724-5.

56. Chertow GM, Pupim LB, Block GA, et al. Evaluation of Cinacalcet therapy to lower cardiovascular events (EVOLVE): rationale and design overview. Clin J Am Soc Nephrol2007; 2:898-905

57. Charytan C, Coburn JW, Chonchol M, et al. Cinacal- 
cethydrochloride is an effective treatment for secondary hyperparathyroidism in patients with CKD not receiving dialysis. Am J Kidney Dis 2005; 46: 58-67.

58. Kestenbaum B, Sampson JN, Rudser KD, et al. Serum phosphate levels and mortality risk among people with chronic kidney disease. J Am Soc Nephrol 2005; 16:520-8.

59. De Francisco ALM, Pinera C, Palomar R. Cinacalcet should be used to treat secondary hyperparathyroidism in stage 3-4 chronic kidney disease. Nat Clin Pract online pub 27 May 2008.

60. Coyne DW. Cinacalcet should not be used to treat secondary hyperparathyroidism in stage 3-4 chronic kidney disease. Nat Clin Pract online pub 13 May 2008.

61. Messa P, Sindici C, Cannella G, et al. Persistent secondary hyperparathyroidism after renal transplantation. Kidney Int 1998; 54:1704-1713

62. Evenepoel P, Claes K, Kuypers D et al. Natural historyof parathyroid function and calcium metabolism after kidney transplantation: a single centre study. Nephrol Dial Transplant2004; 19:1281-1287

63. Gwinner W, Suppa S, Mengel M et al. Early calcification of renal allografts detectedby protocol biopsies: causes and clinical implications. Am J Transplant 2005; 5: 1934-41.
64. Szwarc I, Argilés A, Garrigue V, Delmas S, Chong G, Deleuze S, Mourad G. Cinacalcet chloride is efficient and safe in renal transplant recipients with posttransplant hyperparathyroidism. Transplantation 2006; 82: 675-80.

65. Kruse AE, Eisenberger U, Frey FJ, Mohaupt MG. Effect of cinacalcet cessation in renal transplant recipients with persistent hyperparathyroidism. Nephrol Dial Transplant 2007; 22: 2362-5.

66. Serra AL, Wuhrmann C, Wüthrich RP. Phosphatemic effect of cinacalcet in kidney transplant recipients with persistent hyperparathyroidism. Am J Kidney Dis 2008; 52(6): 1151-7

67. Bergua C, Torregrosa JV, Fuster D, Gutierrez-Dalmau A, Oppenheimer F, Campistol JM. Effect of cinacalcet on hypercalcemia and bone mineral density in renal transplanted patients with secondary hyperparathyroidism. Transplantation 2008; 86: 413-7.

68. Falck P, Vethe NT, Asberg A, Midtvedt K, Bergan S, Reubsaet JL, Holdaas H. Cinacalcet's effect on the pharmacokinetics of tacrolimus, cyclosporine and mycophenolate in renal transplant recipients. Nephrol Dial Transplant 2008; 23: 1048-53. 